\title{
SYSTEMS-DISCONJUGACY OF A FOURTH-ORDER DIFFERENTIAL EQUATION ${ }^{1}$
}

\author{
JOHN H. BARRETT ${ }^{2}$
}

There has been a recent renewal of interest in the oscillatory behavior of solutions of linear self-adjoint differential equations of the fourth order. Leighton and Nehari [5] reopened the study with a new characterization of conjugate points for equations of the type

$$
\left(r(x) y^{\prime \prime}\right)^{\prime \prime}-p(x) y=0,
$$

where $r(x)>0$ and $p(x)$ are both continuous on $[a, \infty)$ and $p(x)$ does not change sign, and related conjugate point properties to oscillation. They gave extensive evidence indicating that the oscillatory behavior when $p(x)$ is positive is essentially different from that when $p(x)$ is negative. Relatively little is known in general when $p(x)$ changes sign or when derivative terms of order less than four are present-except those facts which can be obtained by a simple piecing together of intervals or by the relatively few transformations into the type (1). Earlier study of this subject was done by W. M. Whyburn [8] and others listed in the bibliography of [5].

This paper is primarily concerned with the fourth-order equation (1) where $r(x)$ and $p(x)$ are both positive and continuous on $[a, \infty)^{3}$ and the designation "(1)" will denote the equation (1), together with these restrictions on the coefficients except in one or two cases in which departure from this convention will be explicitly given. For example, in $\$ 3$ there is a theorem insuring the disconjugacy of (1) without regard of the sign, or changes of sign, of $p(x)$.

Leighton and Nehari [5] introduced the double-zero conjugate point concept of which the first conjugate point $\eta_{1}(a)$ of $a$ is defined as follows and is called the first $L N$-conjugate point:

Definition 1. The number $\eta_{1}(a)$ is the smallest number $b \in(a, \infty)$ for which the two point boundary conditions ${ }^{4}$

$$
y(a)=y^{\prime}(a)=y(b)=y^{\prime}(b)=0
$$

are satisfied by a nontrivial solution of (1).

Presented to the Society, November 19, 1960; received by the editors April 13, 1960 and, in revised form, May 23, 1960.

${ }^{1}$ Sponsored by the U. S. Army under Contract No. DA-11-022-ORD-2059.

2 On leave from the University of Utah 1959-60.

'Sufficient for existence of solutions $y(x)$ of (1) such that $y$ and $r y^{\prime \prime} \in C^{\prime \prime}[a, \infty)$.

${ }^{4}$ For standard names for boundary conditions (2), (3), (5), (6) see R. Courant and D. Hilbert, Methods of mathematical physics, Vol. 1, New York, Interscience, 1953, pp. 295-296. 
In [2], the author introduced a companion number $\mu_{1}(a)$ which corresponds to the focal point conditions for second-order equations.

Definition 2. The number $\mu_{1}(a)$ is the smallest number $b \in(a, \infty)$ for which

$$
y(a)=y^{\prime}(a)=y_{1}(b)=y_{1}^{\prime}(b)=0
$$

is satisfied nontrivially by (1). (The notation $y_{1}(x)=r(x) y^{\prime \prime}(x)$ will be used throughout this paper.)

If $\eta_{1}(a)$ does not exist then no solution of (1) has more than three zeros on $[a, \infty)$ [5] and equation (1) is said to be $L N$-disconjugate [2]. The reader is referred to the above-mentioned references for the relation of these numbers to the oscillatory behavior of solutions of (1).

In this paper, the fourth-order equation (1) is expressed in an obvious way as a system of two second order equations in the vectormatrix form. ${ }^{5}$

$$
\alpha^{\prime \prime}-A(x) \alpha=0, \quad \alpha=\left(\begin{array}{c}
y \\
y_{1}
\end{array}\right), \quad A=\left(\begin{array}{cc}
0 & 1 / r \\
p & 0
\end{array}\right)
$$

and conjugate points are defined to be the well-known ones for systems (4) and are called systems-conjugate points of (1):

Definition 3. The number $\hat{\eta}_{1}(a)$ is the smallest $b \in(a, \infty)$ for which

$$
y(a)=y_{1}(a)=y(b)=y_{1}(b)=0
$$

is satisfied nontrivially by (1).

Definition 4 . The number $\hat{\mu}_{1}(a)$ is similarly determined by

$$
y(a)=y_{1}(a)=y^{\prime}(b)=y_{1}^{\prime}(b)=0 .
$$

For any of these numbers it is easy to show that if one such number $b$ exists then there is a smallest on $(a, \infty)$. If $\hat{\eta}_{1}(a)$ does not exist then equation (1) is said to be systems-disconjugate on $[a, \infty)$. It will be seen in $\S 4$ that the existence of $\eta_{1}(a)$ implies that of $\hat{\eta}_{1}(a)$, so that systems-disconjugacy is a subclass of $L N$-disconjugacy. Examples are given to show the converse is not true. If infinitely many systemsconjugate points $\left\{\hat{\eta}_{n}(a)\right\}$ exist it will be seen that equation (1) is oscillatory, but the existence of $\hat{\eta}_{1}(b)$ for each $b \in[a, \infty)$ does not imply oscillation-thus departing again from the properties of the $L N$-conjugate points.

An oscillation theorem and a disconjugacy theorem for equation (1), with $p r=1$, are established which depend on the oscillation of a

\footnotetext{
- Another second-order systems representation is utilized in [7].
} 
second order equation. This disconjugacy theorem is then applied to give a disconjugacy theorem for the more general equation (1).

Extensive use will be made of the sequence $\{U, V, u, v\}$ of fundamental solutions of (1) determined by the identity matrix Wronskian at $x=a$ :

$$
\begin{aligned}
& y(a) \quad y^{\prime}(a) \quad y_{1}(a) \quad y_{1}^{\prime}(a) \\
& \begin{array}{l}
y=U: \\
y=V: \\
y=u: \\
y=v:
\end{array} \quad\left(\begin{array}{llll}
1 & 0 & 0 & 0 \\
0 & 1 & 0 & 0 \\
0 & 0 & 1 & 0 \\
0 & 0 & 0 & 1
\end{array}\right) .
\end{aligned}
$$

1. Relations between systems-conjugacy and focal conditions. It was seen in [2] that the existence of $\eta_{1}(a)$ implies that of $\mu_{1}(a)$ but the converse is true only under additional conditions (e.g., $\int{ }^{\infty} 1 / r=\infty$ ), as is the case for second-order real equations [1]. However, the systems concepts are more closely related.

THEOREM 1.1. The first systems conjugate point $\hat{\eta}_{1}(a)$ exists if, and only if, the number $\hat{\mu}_{1}(a)$ exists. Furthermore, $a<\hat{\mu}_{1}(a)<\hat{\eta}_{1}(a)$.

Proof. Note that $\hat{\eta}_{1}(a)$ and $\hat{\mu}_{1}(a)$ are the first zeros on $(a, \infty)$ of $\hat{\sigma}^{\prime}$ and $\hat{\tau}^{\prime}$, respectively, where

(8) $\hat{\sigma}=V v^{\prime}-v V^{\prime}, \hat{\rho}=V_{1} v_{1}^{\prime}-v_{1} V_{1}^{\prime}, \hat{\tau}=V^{\prime} v_{1}-v^{\prime} V_{1}\left(\equiv V v_{1}^{\prime}-v V_{1}^{\prime}\right)$.

The following identities will be helpful (see [2] for similar ones):

$$
\hat{\rho}^{\prime}=-p r \hat{\sigma}^{\prime}, \quad\left(r \hat{\sigma}^{\prime}\right)^{\prime}=2 \hat{\tau}, \quad \hat{\tau}^{\prime \prime}=\frac{\hat{\rho}}{r}-p \hat{\sigma},
$$

as well as the initial values:

$$
\begin{aligned}
& \hat{\sigma}(a)=\hat{\sigma}^{\prime}(a)=\left(r \hat{\sigma}^{\prime}\right)^{\prime}(a)=0, \quad\left(r \hat{\sigma}^{\prime}\right)^{\prime \prime}(a)=2, \quad \hat{\tau}(a)=0, \\
& \hat{\rho}(a)=\hat{\rho}^{\prime}(a)=\left(\hat{\rho}^{\prime} / p\right)^{\prime}(a)=0, \quad\left(\hat{\rho}^{\prime} / p\right)^{\prime \prime}(a)=-2, \quad \hat{\imath}^{\prime}(a)=1 .
\end{aligned}
$$

CASE 1. Suppose $\hat{\eta}_{1}(a)$ exists. Then $\hat{\rho}\left(\hat{\eta}_{1}\right)=0=\hat{\rho}^{\prime}(a)$ and by Rolle's Theorem there is a zero of $\hat{\tau}(x)$, and hence one of $\hat{\tau}^{\prime}(x)$, on $\left(a, \hat{\eta}_{1}\right)$. Therefore, $\hat{\mu}_{1}(a)$ exists and one part of the theorem is proved.

CASE 2. Suppose $\hat{\mu}_{1}(a)$ exists and $\hat{\eta}_{1}(a)$ does not exist. Then $\hat{\sigma}^{\prime}>0$ and, hence, $\hat{\sigma}>0$ on $(a, \infty)$. Also, $\hat{\rho}^{\prime}<0$ and $\hat{\rho}<0$ on $(a, \infty)$. Since $(\hat{\tau} / V)^{\prime}=-\hat{\sigma} V_{1}^{\prime} / V^{2}<0$ then $\hat{\tau}(x)$ has at most one zero on $(a, \infty)$. The facts that $\hat{\tau}(x)$ is a solution of the second order differential equation 


$$
\left(\frac{\hat{\imath}^{\prime}}{\left(V^{\prime} V_{1}^{\prime}\right)^{1 / 2}}\right)^{\prime}+\frac{1}{2\left(V^{\prime} V_{1}^{\prime}\right)^{1 / 2}}\left(\frac{V_{1}^{\prime}}{v V^{\prime}}+\frac{p V^{\prime}}{V_{1}^{\prime}}\right) \hat{\imath}=0
$$

and that $\hat{\tau}^{\prime}\left(\hat{\mu}_{1}\right)=0$ yield [2] that $\hat{\tau}(x)$ has a zero $x=t_{1}$ and that $x=\hat{\mu}_{1}$ is the only zero of $\hat{\tau}^{\prime}(x)$ on $(a, \infty)$.

As in [2], consider the positive quotients on $(a, \infty)$ :

$$
\lambda_{0}=V / v, \quad \lambda_{1}=V^{\prime} / v^{\prime}, \quad \lambda_{2}=V_{1} / v_{1}, \quad \lambda_{3}=V_{1}^{\prime} / v_{1}^{\prime} .
$$

Analysis of their monotone properties and differences (as in [2]) yields that there exists a positive constant $\lambda^{*}$ such that on $\left(t_{1}, \infty\right)$ the solution $y(x)=V(x)-\lambda^{*} v(x)$ satisfies $y>0, y^{\prime}<0$ and $y^{\prime \prime}<0$, which is contradictory. Therefore, the existence of $\hat{\mu}_{1}(a)$ yields the existence of $\hat{\eta}_{1}(a)$, without further restrictions on the coefficient of (1).

Similar analysis establishes that $\sigma^{\prime}>0$ on $(a, \infty)$ if, and only if, $\tau^{\prime}>0$ on $(a, \infty)$, where $\sigma=u v^{\prime}-v u^{\prime}$ and $\tau=u^{\prime} v_{1}-v^{\prime} u_{1}$ (see §4). Also,

Lemma 1.1. If $\hat{\eta}_{1}(a)$ does not exist (i.e., equation (1) is systemsdisconjugate) then there is a positive number $\lambda$ such that on $(a, \infty)$ the solution $y=V-\lambda v$ satisfies $y>0, y^{\prime}>0, y_{1}<0$ and $y_{1}^{\prime}<0$.

Suppose that $\hat{\eta}_{1}(a)$ does not exist but that there is a number $b \in(a, \infty)$ such that $\hat{\eta}_{1}(b)$ exists. By definition, there is a nontrivial solution $z(x)$ such that $z(b)=z_{1}(b)=z^{\prime}(c)=z_{1}^{\prime}(c)=0, c=\hat{\mu}_{1}(a)$ (exists by Theorem 1.1). If $z^{\prime}(b)>0$ then $z_{1}^{\prime}(b)<0$ and it is easily determined that $z(c)>0$ and $z_{1}(c)<0$. Using the solution $y(x)$ of Lemma 1.1 and recalling that $[5]$

$$
S[y ; z]=y z_{1}^{\prime}-z y_{1}^{\prime}-y^{\prime} z_{1}+z^{\prime} y_{1} \equiv \text { constant }
$$

a contradiction in signs at $x=b$ and $x=c$ is achieved.

Lemma 1.2. If $\hat{\eta}_{1}(a)$ does not exist then $\hat{\eta}_{1}(b)$ does not exist for $b \in(a, \infty)$.

Note that $y^{\prime}>0, y_{1}<0$ and $y_{1}^{\prime}<0$ on $(a, \infty)$ are contradictory if $\int^{\infty} 1 / r=\infty$ and $y_{1}^{\prime}<0, y>0, y^{\prime}>0$ cannot be true if $\int^{\infty} p=\infty$. Hence:

THEOREM 1.2. If either $\int^{\infty} 1 / r=\infty$ or $\int^{\infty} p=\infty$ then $\hat{\eta}_{1}(a)$ exists, in fact, $\hat{\eta}_{1}(b)$ exists for all $b \in[a, \infty)$.

Recall from [2] that the hypothesis of Theorem 1.2 is not sufficient for the existence of either the first $L N$-conjugate point $\eta_{1}(a)$ or $\mu_{1}(a)$. However, $\int^{\infty} 1 / r=\infty$ and $\int^{\infty} p=\infty$ is sufficient for oscillation (i.e., there is a nontrivial solution of (1) with infinitely many zeros on $(a, \infty))$. 
Corollary 1.2.1. If $r(x)$ is positive and continuous on $(a, \infty)$ then every equation of the form

$$
\left(r(x) y^{\prime \prime}\right)^{\prime \prime}-r(x) y=0
$$

is systems-conjugate and for each $b \in[a, \infty)$ the number $\hat{\eta}_{1}(b)$ exists.

It should be noted here that for $L N$-conjugate points, if for each $b \in(a, \infty)$ there exists $\eta_{1}(b)$ then equation (1) is oscillatory [5]. That this is not so for systems-conjugate points is shown by the following example.

EXAMPLE 1.1. $p=r=e^{-2 x}, a=0$, in (1) gives

$$
\left(e^{-2 x} y^{\prime \prime}\right)^{\prime \prime}-e^{-2 x y}=0 .
$$

If $y(x)$ is a solution of (12) then $z(x)=e^{-x} y(x)$ is a solution of $z^{i v}-2 z^{\prime \prime}$ $=0$ and $z(x)$, hence $y(x)$, can have at most three zeros, i.e., is $L N$ disconjugate and $\eta_{1}(a)$ does not exist. However, $\int^{\infty} 1 / r=\int^{\infty} e^{2 x}=\infty$ and $\hat{\eta}_{1}(a)$ exists by Theorem 1.2. This example was quoted in [2] as a fourth-order equation where $\mu_{1}(a)$ does not exist. Furthermore, the conclusion of Theorem 1.2 for $L N$-disconjugate points would insure oscillation of (1), which obviously is not so for (12). This example will be investigated further in $\$ 4$.

2. A special case. In equation (1) let $q(x)=p(x)=1 / r(x)$, continuous and positive on $[a, \infty)$, and obtain the special case

$$
\left(y^{\prime \prime} / q(x)\right)^{\prime \prime}-q(x) y=0 .
$$

As in $[1 ; 2]$ define the reciprocal equation of (1) to be

$$
\left(y^{\prime \prime} / p(x)\right)^{\prime \prime}-y / r(x)=0
$$

and note that (13) is its own reciprocal. Furthermore the system

$$
\alpha^{\prime \prime}-A(x) \alpha=0
$$

is further simplified so that

$$
A(x)=q(x)\left(\begin{array}{ll}
0 & 1 \\
1 & 0
\end{array}\right)
$$

and is symmetric.

Lemma 2.1. If $U^{*}, V^{*}, u^{*}, v^{*}$ is the fundamental sequence of solutions of $\left(1^{*}\right)$ satisfying the identity Wronskian at $x=a$ then

$$
U_{1} \equiv u^{*}, \quad V_{1} \equiv v^{*}, \quad u_{1} \equiv U^{*}, \quad v_{1} \equiv V^{*},
$$


and for equation (13) these identities specialize to

$$
U_{1} \equiv u, \quad V_{1} \equiv v, \quad u_{1} \equiv U, \quad v_{1} \equiv V .
$$

Lemma 2.2 .

$$
Y(x)=\left(\begin{array}{ll}
V(x) & v(x) \\
v(x) & V(x)
\end{array}\right)
$$

is a fundamental matrix solution of (4) related to (13), in fact,

$$
Y^{\prime \prime}=A Y, \quad Y(a)=0, \quad Y^{\prime}(a)=E .
$$

Lemma 2.3. The first systems conjugate points, $\hat{\eta}_{1}(a)$, is the first zero on $(a, \infty)$ of $\operatorname{det}(Y)=V^{2}-v^{2}=\hat{\sigma}^{\prime} / q$ (i.e., the first zero of $V(x)-v(x)$ ).

Lemma 2.4. The difference $z(x)=V(x)-v(x)$ satisfies the secondorder equation and initial conditions

$$
z^{\prime \prime}+q(x) z=0, \quad z(a)=0, \quad z^{\prime}(a)=1 .
$$

From these results it follows immediately that

Theorem 2.1. In order for the fourth-order equation (13) to be systems-disconjugate it is necessary and sufficient that the second-order equation (15) be disconjugate on $(a, \infty)$.

Therefore, one may use the known theory for disconjugacy of second order equations $(3)$, e.g. $[1 ; 4 ; 6]$, to establish analogous properties of the fourth order case (13). For example, Lemma 2.4 and the monotonicity result of Hille [4] for (15) readily verifies Theorem 1.1 for the special case (13).

The observation that the general solution of (13) is given by the sum of the general solutions of $y^{\prime \prime}+q(x) y=0$ and $y^{\prime \prime}-q(x) y=0$, together with Theorem 2.1, yields the oscillation criterion:

THEOREM 2.2. The special fourth-order equation (13) is oscillatory if, and only if, the second-order equation (15) is oscillatory.

EXAMPLE 2.1. $q(x)=k / 4 x^{2}, k>0$ and $a=1$ in equation (13) yields

$$
\left(\frac{4 x^{2}}{k} y^{\prime \prime}\right)^{\prime \prime}-\frac{k}{4 x^{2}} y=0,
$$

with $\int^{\infty} 1 / r<\infty$ and $\int^{\infty} p<\infty$. Since $z^{\prime \prime}+\left(1 / 4 x^{2}\right) z=0$ is disconjugate on $(1, \infty)$ then $\left(16_{k}\right)$ with $k \leqq 1$ is systems-disconjugate, i.e., $\hat{\eta}_{1}(a)$ and $\hat{\mu}_{1}(a)$ do not exist. For $k>1$, equation $\left(16_{k}\right)$ is oscillatory. This example will be further investigated in $\$ 4$. 
3. Applications of the special case. Return to the more general system (4) with coefficient matrix

$$
A=\left(\begin{array}{ll}
0 & 1 / r \\
p & 0
\end{array}\right)
$$

The symmetric part of a matrix $A$ is defined [3] as $A_{0}=\left(A+A^{T}\right) / 2$ and in this case turns out to be

$$
A_{0}=\frac{1}{2}\left(\frac{1}{r}+p\right)\left(\begin{array}{ll}
0 & 1 \\
1 & 0
\end{array}\right)=q_{0}(x)\left(\begin{array}{ll}
0, & 1 \\
1 & 0
\end{array}\right) .
$$

According to Hartmann and Wintner [3], if the symmetric equation

$$
\alpha^{\prime \prime}-A_{0} \alpha=0
$$

is disconjugate on $(a, \infty)$ then so is equation (4). But by Lemmas 2.2 and 2.3 equation (4) is disconjugate if, and only if, $\operatorname{det}\left(Y_{0}\right)=V_{0}^{2}-v_{0}^{2}$ is nonzero on $(a, \infty)$ where $Y_{0}, V_{0}, v_{0}$ are the quantities of the preceding section for the special equation $\left(4_{0}\right)$. By noting that $V_{0}-v_{0}$ and $V_{0}+v_{0}$ satisfy, respectively, the second-order equations:

$$
z^{\prime \prime}+q_{0}(x) z=0 \text { and } z^{\prime \prime}-q_{0}(x) z=0
$$

it follows readily that

THEOREM 3.1. If there exists a positive constant $k$ such that for $q(x)=(k / r+p / k) / 2$, both second-order equations $z^{\prime \prime}+q(x) z=0$ and $\boldsymbol{z}^{\prime \prime}-q(x) z=0$ are disconjugate on $[a, \infty)$ then the fourth-order equation (1) is systems-disconjugate on $[a, \infty)$ and, hence, LN-disconjugate regardless of the sign of $p(x)$. (Of course, if $p>0$ then $z^{\prime \prime}-q z=0$ is automatically disconjugate.)

4. Comparison of conjugate points. Recall from [2] that $\eta_{1}(a)$ and $\mu_{1}(a)$ are the first zeros on $(a, \infty)$ of, respectively, $\sigma(x)$ and $\rho(x)$ where (recalling (7))

$$
\sigma=u v^{\prime}-v u^{\prime}, \rho=u_{1} v_{1}^{\prime}-v_{1} u_{1}^{\prime}, \tau=u^{\prime} v_{1}-v^{\prime} u_{1}\left(\equiv u v_{1}^{\prime}-v u_{1}^{\prime}\right) .
$$

Also the fact that if $y$ and $z$ are solutions of (1) then

$$
S[y ; z]=y z_{1}^{\prime}-z y_{1}^{\prime}-y^{\prime} z_{1}+z^{\prime} y_{1} \equiv \text { constant }
$$

yields that both $\sigma(x)$ and $\hat{\sigma}(x)$ are linearly independent solutions of

$$
\left(\frac{r \sigma^{\prime}}{v^{2}}\right)^{\prime}+\frac{2 v_{1}}{v^{3}} \sigma=0 \text { on }(a, \infty) \text {. }
$$


Since $\sigma(a)=\sigma^{\prime}(a)=\left(r \sigma^{\prime}\right)^{\prime}(a)=\left(r \sigma^{\prime}\right)^{\prime \prime}(a)=0,\left(r \sigma^{\prime}\right)^{\prime \prime \prime}(a)>0$ then at the regular singular point $x=a$ there is a higher order zero of $\sigma(x)$ than of $\hat{\sigma}(x)$ and the Wronskian

$$
\hat{\sigma} \sigma^{\prime}-\hat{\sigma}^{\prime} \sigma=\frac{k v^{2}}{r} \operatorname{gives} \sigma(x)=k \hat{\sigma}(x) \int_{a}^{x} \frac{v^{2}}{r \hat{\sigma}^{2}}, \quad \text { if } \hat{\sigma} \neq 0 \text { on }(a, \infty) \text {. }
$$

Therefore, a zero of $\sigma(x)$ on $(a, \infty)$ yields a zero of $\hat{\sigma}(x)$ and, hence, one of $\hat{\sigma}^{\prime}(x)$.

THEOREM 4.1. If $\eta_{1}(a)$ exists then so does $\hat{\eta}_{1}(a)$ and $\hat{\mu}_{1}(a)$.

The hypothesis of Theorem 4.1 cannot be weakened to the existence of $\mu_{1}(a)$ as is seen by further study of Example 2.1. As has already been seen, $\hat{\eta}_{1}(a)$ does not exist. Furthermore, if $y(x)$ is a solution of (161) $\left(4 x^{2} y^{\prime \prime}\right)^{\prime \prime}-\left(1 / 4 x^{2}\right) y=0$ and $t=(\ln x) / 2^{1 / 2}$, then

$$
H(t)=e^{-t / 2^{1 / 2}} y\left(e^{2^{1 / 2} t}\right) \text { is a solution of } H^{(\mathrm{i} v)}-\ddot{B}=0 .
$$

Hence no solution of (16) can have more than 3 zeros on $[1, \infty)$ and $\eta_{1}(1)$ does not exist. For this simple case $\rho^{\prime}=-\sigma^{\prime}$ yielding $\rho(x)=1$ $-\sigma(x), \quad v(x)=2^{3 / 2} e^{t / 2^{1 / 2}} H(t)$ and $u(x)=e^{t / 2^{1 / 2}}\left[2 \dot{H}-2^{1 / 2} H\right]$, where $H(t)=\sinh t-t$ and $t=(\ln x) / 2^{1 / 2}$. Now,

$$
\sigma(x)=4\left[(\dot{H})^{2}-H \ddot{H}\right]=2(1-\cosh t)+t \sinh t \rightarrow \infty \text { as } x \rightarrow \infty \text {. }
$$

Therefore $\rho(1)=1$ and $\rho(x) \rightarrow-\infty$ as $x \rightarrow \infty$ and $\mu_{1}(1)$ exists, but there are no other zeros of $\rho(x)$ on $(1, \infty)$ since $\hat{\sigma}^{\prime}>0$ on $(a, \infty)$ implies $\sigma^{\prime}>0$ on $(a, \infty)$.

Furthermore, the converse of Theorem 4.1 is not true, as was seen by Example 1.1. However, separation theorems applied to (19) yield:

THEOREM 4.2. If $\hat{\eta}_{1}(a), \hat{\eta}_{2}(a)$ and $\hat{\eta}_{3}(a)$ (first 3 zeros of $\hat{\sigma}^{\prime}$ ) all exist then $\eta_{1}(a)$ exists and if $\hat{\sigma}^{\prime}(x)$ has infinitely many zeros on $(a, \infty)$ then so does $\sigma(x)$ and equation (1) is oscillatory.

Return again to Example 1.1:

$$
\left(e^{-2 x} y^{\prime \prime}\right)^{\prime \prime}-e^{-2 x} y=0, \quad a=0 .
$$

The fundamental solutions $v(x)=\left(e^{x} / 2\right) h(x)$, where $h(x)$ $=\left(\sinh 2^{1 / 2} x-2^{1 / 2} x\right) / 2^{1 / 2} . \quad$ Furthermore, $\quad V^{\prime \prime}(x)=v(x), \quad V(x)$ $=e^{x}\left[-h^{\prime}+3 h / 2+x\right]$ and $v^{\prime \prime}(x)=e^{x}\left[h^{\prime}+3 h / 2+x\right]$. Now, $e^{-2 x} \hat{\sigma}^{\prime}=2 h^{2}$ $-\left(h^{\prime}\right)^{2}+3 h x+x^{2} \sim-2^{-3 / 2} x e^{2^{1 / 2} x}$ as $x \rightarrow \infty$. Also, $\left(e^{-2 x} \hat{\sigma}^{\prime}\right)^{\prime \prime \prime}=2 \hat{\tau}^{\prime \prime}$ $=-2 x(h+x)<0$ on $(0, \infty)$ and $\hat{\sigma}^{\prime}$ has at most one zero on $(0, \infty)$. Therefore, $\hat{\eta}_{1}(0)$ exists but $\hat{\eta}_{2}(0)$ does not. Finally, as was observed in [2], $\rho(x)>0$ on $(0, \infty)$ and $\mu_{1}(0)$ does not exist, and even a weaker converse of Theorem 4.1 is not true. 
It would be interesting to know what conditions added to the existence of $\hat{\eta}_{1}(a)$ would imply the existence of $\eta_{1}(a)$ or $\mu_{1}(a)$.

\section{BIBLIOGRAPHY}

1. J. H. Barrett, Disconjugacy of second-order linear differential equations with nonnegative coefficients, Proc. Amer. Math. Soc. vol. 10 (1959) pp. 552-561.

2. - Disconjugacy of a self-adjoint differential equation of the fourth order, Chapter 1, OOR Technical Report, Utah (1959), pp. 1-24; Pacific J. Math. (to appear).

3. P. Hartman and A. Wintner, On disconjugate differential systems, Canad. J. Math. vol. 8 (1956) pp. 72-81.

4. E. Hille, Nonoscillation theorems, Trans. Amer. Math. Soc. vol. 64 (1948) pp. 234-252.

5. W. Leighton and Z. Nehari, On the oscillation of solutions of self-adjoint linear differential equations of the fourth order, Trans. Amer. Math. Soc. vol. 89 (1958) pp. 325-377. (Contains an extensive bibliography.)

6. Z. Nehari, Oscillation criteria for second-order linear differential equations, Trans. Amer. Math. Soc. vol. 85 (1958) pp. 428-445.

7. H. M. Sternberg and R. L. Sternberg, $A$ two-point boundary problem for ordinary selfadjoint differential equations of fourth order, Canad. J. Math. vol. 6 (1954) pp. 416419.

8. W. M. Whyburn, On self-adjoint ordinary differential equations of the fourth order, Amer. J. Math. vol. 52 (1930) pp. 171-196.

Mathematics Research Center, U. S. Army, Madison, Wisconsin 